\title{
Measurement of end-tidal carbon dioxide concentration during cardiopulmonary resuscitation
}

\author{
D. J. STEEDMAN \& C. E. ROBERTSON \\ Department of Emergency Medicine, Royal Infirmary, Lauriston Place, Edinburgh
}

\section{SUMMARY}

End-tidal carbon dioxide concentrations were measured prospectively in 12 cardiac arrest patients undergoing cardiopulmonary resuscitation (CPR) in an accident and emergency department.

The end-tidal carbon dioxide $\left(\mathrm{CO}_{2}\right)$ concentration decreased from a mean ( \pm SD) of $4.55 \pm 0.88 \% 1 \mathrm{~min}$ after chest compression and ventilation was established, to values ranging from $2.29 \pm 0.84 \%$ at $2 \mathrm{~min}$ to $1.56 \pm 0.66 \%$ following $8 \mathrm{~min}$ of CPR. Spontaneous circulation was restored in five patients. This was accompanied by a rapid rise in end-tidal $\mathrm{CO}_{2}$ which peaked at $2 \mathrm{~min}(3.7 \pm 1.08 \%)$. Changes in end-tidal $\mathrm{CO}_{2}$ values were often the first indication of return of spontaneous cardiac output.

There was a significant difference in the end-tidal $\mathrm{CO}_{2}$ in patients undergoing CPR before return of spontaneous circulation $(2.63 \pm 0.32 \%)$ and patients who failed to develop spontaneous output $(1.64 \pm 0.89 \%)(p<0.001)$.

We conclude that measurement of end-tidal $\mathrm{CO}_{2}$ concentration provides a simple and non-invasive method of measuring blood flow during CPR and can indicate return of spontaneous circulation.

\section{INTRODUCTION}

Difficulties in monitoring the effectiveness of efforts at resuscitation have recently been highlighted (Skinner, 1989). Palpation of the carotid or femoral pulse is the usual method of monitoring circulation but may not represent forward flow. Most studies investigating blood flow during external cardiac massage have used invasive techniques in animal preparations. The relevance of animal studies to resuscitation

Correspondence: D. J. Steedman, Senior Registrar, Department of Emergency Medicine, Royal Infirmary, Lauriston Place, Edinburgh EH3 9YW, U.K. 
in humans has been questioned, and invasive monitoring is usually impracticable in the emergency situation (Chamberlain et al., 1987). A non-invasive monitor which reflects blood flow during precordial compression would not only provide useful clinical tool, but facilitate clinical trials in humans to evaluate the effect of changes in CPR protocols.

End-tidal carbon dioxide concentration has been shown to correlate with cardia $\varepsilon_{0}^{2}$ output and coronary blood flow in experimental animals during cardiopulmonary resuscitation (Sanders et al., 1985; Weil et al., 1985). Subsequently, in critically il patients, measurement of end-tidal $\mathrm{CO}_{2}$ provided a practical and non-invasive method for monitoring blood flow generated by precordial compression (Falk et al., 1988). Clearly, caution is required before extrapolating from studies carriedout in an intensive care environment to the more common situation of unexpected cardiac arrest.

The aim of this study was to assess the clinical applicability of end-tidal carbon dioxide measurements in patients undergoing cardiopulmonary resuscitation in an accident and emergency department.

\section{SUBJECTS AND METHODS}

Adults with non-traumatic, normothermic cardiac arrest, admitted to the Acciden and Emergency Department of the Edinburgh Royal Infirmary were studied. patients had sustained cardiorespiratory arrest in the pre-hospital setting and hadp received only basic life support prior to arrival. On admission, patients weite treated according to the guidelines of the UK Resuscitation Council (1984). Electricas DC counter shock and anti-arrhythmic agents were used as appropriate. All patients were intubated and ventilated with $100 \%$ oxygen and mechanical chest com pression was administered using a Michigan Instruments Thumper, Model $1004 \overrightarrow{\vec{*}}$ functioning at a ratio of 5 chest compressions to one ventilation (Little et al., 1974) $\frac{3}{3}$ CPR was performed with sufficient force to compress the chest 2.5-3 inches? $(80-100 \mathrm{lbs})$ and peak inspiratory pressure was set at $30 \mathrm{cms}$ of $\mathrm{H}_{2} \mathrm{O}$. During CPR four patients were given $8.4 \%$ sodium bicarbonate at a mean dose of $100 \mathrm{mEq}$ between time 3 and $16 \mathrm{~min}$ (mean $8.4 \mathrm{~min}$ ).

The concentration of expired carbon dioxide was measured continuously with an infra-red absorption carbon dioxide analyser (Model 5200, Ohmeda, Louisville, USA) calibrated to a $5 \%$ carbon dioxide standard producing an accuracy of $\pm 1.5 \mathrm{mmHg}$ (from $0-75 \mathrm{mmHg}$ ). The $\mathrm{CO}_{2}$ values were automatically compensated for pressure and temperature variations. The sampling adaptor was sited betweer the endotracheal tube and the ventilatory circuit tubing.

All end-tidal $\mathrm{CO}_{2}$ values are reported as the percentage of carbon dioxide in ${ }^{N}$ exhaled gas (mean $\pm \mathrm{SD}$ ). Data were analysed using a two tailed t-test for paired: and unpaired data and differences considered significant when $\mathrm{P}<0.05$. 


\section{RESULTS}

The study population comprised 8 males and 4 females with a mean age of $69 \pm 17$ years. The presenting dysrrhythmia was asytole in five patients, ventricular fibrillation in five patients and two patients had electro-mechanical dissociation. Five patients developed return of spontaneous circulation, four of whom were discharged from the Accident and Emergency Department (Table 1). The end-tidal $\mathrm{CO}_{2}$ concentration after $1 \mathrm{~min}$ of CPR was $4.55 \pm 0.88 \%$, but declined to $2.29 \pm$ $0.84 \%$ at $2 \mathrm{~min}, 2.25 \pm 1.03 \%$ at $4 \mathrm{~min}$ and $1.66 \pm 0.56 \%$ at $8 \mathrm{~min}$ or more.

Coincident with return of spontaneous circulation in five patients, the end-tidal $\mathrm{CO}_{2}$ significantly increased from $1.8 \pm 0.92 \%$ to $3.38 \pm 1.06 \%$ within one min $(\mathrm{P}<0.05)$. After peaking at $2 \mathrm{~min}(3.7 \pm 1.08 \%)$, the end-tidal $\mathrm{CO}_{2}$ declined to 3.65 $\pm 1.43 \%$ at $4 \mathrm{~min}$ and $3.02 \pm 0.52 \%$ at $8 \mathrm{~min}$ in these patients.

In patients given sodium bicarbonate, the end-tidal $\mathrm{CO}_{2}$ concentration increased from $1.5 \pm 0.49 \%$ to $1.8 \pm 0.54 \%$ but this rise does not reach statistical significance. The difference between the end-tidal $\mathrm{CO}_{2}$ concentration in patients without return of spontaneous circulation $(\mathrm{N}=7,1.64 \pm 0.89 \%)$ and the end-tidal concentration in patients prior to regaining spontaneous output $(\mathrm{N}=5,2.63 \pm 0.32 \%)$ was significant $(\mathrm{P}<0.001)$. Examples of end-tidal $\mathrm{CO}_{2}$ recordings in three cases are illustrated in Figs. 1 and 2.

\section{DISCUSSION}

End-tidal carbon dioxide concentration is dependent on carbon dioxide production, alveolar ventilation and pulmonary blood flow (Powles \& Campbell, 1978). If alveolar ventilation is held constant and carbon dioxide production is assumed to

Table 1. Clinical data in 12 patients. VF denotes ventricular fibrillation and EMD electromechanical dissociation. Four patients received $8 \cdot 4 \%$ sodium bicarbonate during CPR.

\begin{tabular}{lcclccc}
\hline Case & Age & Sex & $\begin{array}{l}\text { Primary } \\
\text { rhythm }\end{array}$ & $\begin{array}{c}\text { Duration } \\
\text { of CPR } \\
\text { (Minutes) }\end{array}$ & $\begin{array}{c}\text { Na HCO } \\
\text { Administered } \\
\text { (mEq) }\end{array}$ & $\begin{array}{c}\text { Discharged } \\
\text { from A\&E }\end{array}$ \\
\hline MB & 69 & F & VF & 20 & - & No \\
WF & 68 & M & Asystole & 11 & 50 & No \\
CD & 75 & F & Asystole & 10 & - & No \\
WS & 68 & M & Asystole & 5 & - & No \\
JA & 73 & M & VF & 14 & - & No \\
HL & 70 & F & EMD & 31 & 200 & No \\
UM & 69 & M & Asystole & 6 & - & Yes \\
PC & 65 & M & Asystole & 5 & - & Yes \\
JA & 78 & M & VF & 9 & - & Yes \\
HF & 50 & F & VF & 3 & 100 & No \\
JL & 68 & M & VF & 12 & - & Yes \\
SA & 19 & M & EMD & 8 & & \\
\hline
\end{tabular}




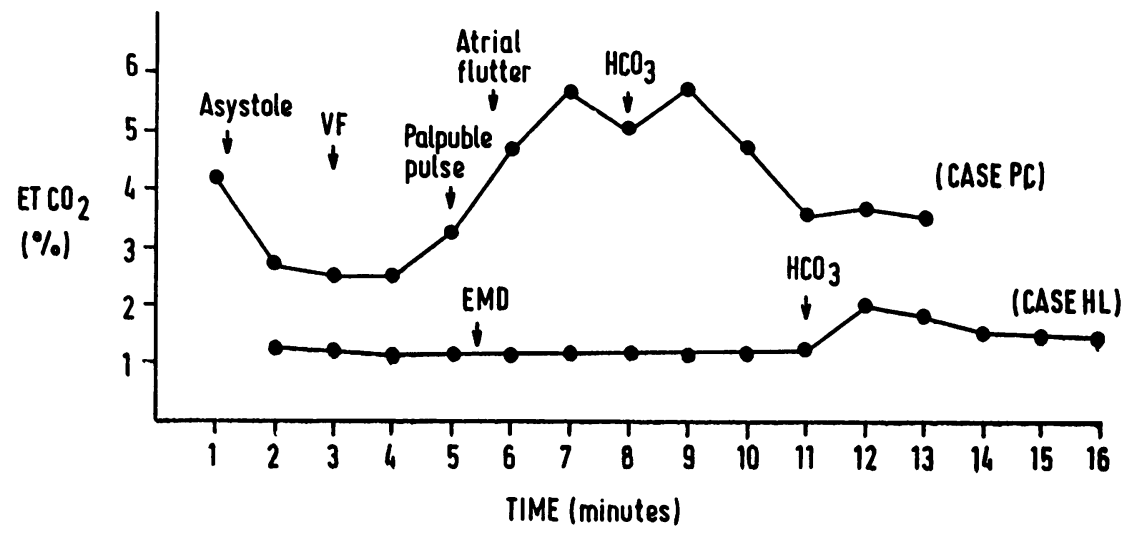

Fig. 1. End-tidal $\mathrm{CO}_{2}$ concentration during resuscitation. Case PC and $\mathrm{HL}$.

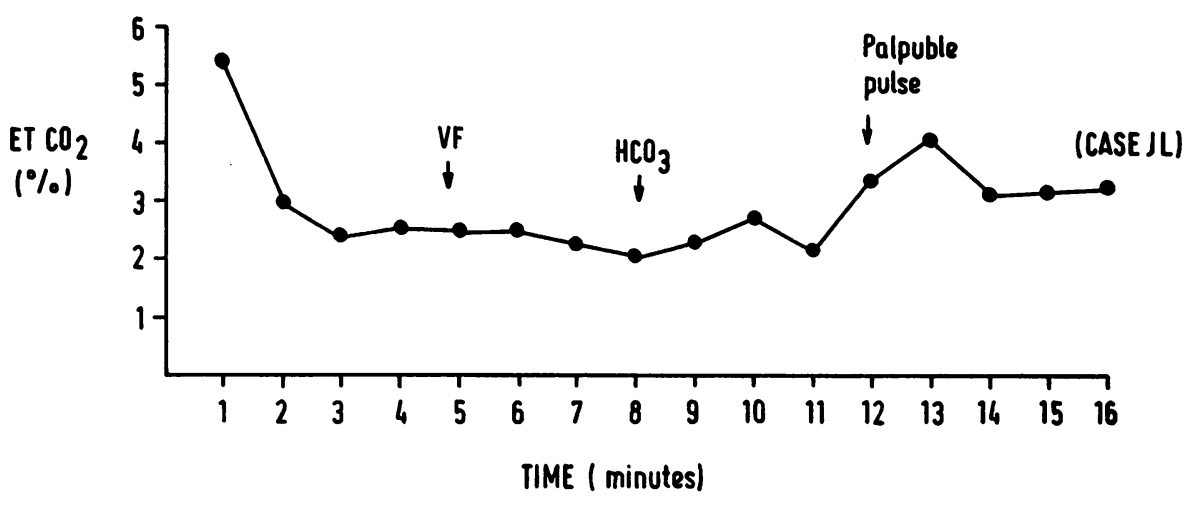

Fig. 2. End-tidal $\mathrm{CO}_{2}$ concentration during resuscitation. Case JL.

be constant, end-tidal $\mathrm{CO}_{2}$ values reflect changes in systemic and pulmonary perfusion. The return of spontaneous circulation in this study was associated with a two-fold increase in the end-tidal $\mathrm{CO}_{2}$ despite constant mechanical ventilation. The low end-tidal $\mathrm{CO}_{2}$ values demonstrated during precordial compression, reflect the poor forward blood flow achieved with the closed technique. If delivery of 3 $\mathrm{CO}_{2}$ to the lungs falls as a result of diminished pulmonary perfusion, $\mathrm{CO}_{2}$ excretion necessarily decreases, even if ventilation is adequate. Closed chest CPR results in reduction of cardiac output to about $25-30 \%$ of pre-arrest values (Del Guercioet al., 1965) and therefore the low pulmonary blood flow becomes the rate limiting? factor in $\mathrm{CO}_{2}$ elimination. If spontaneous circulation returns, or if the pulmonary $\mathrm{C}^{\mathrm{\omega}}$ blood flow increases during $\mathrm{CPR}, \mathrm{CO}_{2}$ delivery to the lungs increases, ventilation perfusion mismatch decreases and the end-tidal $\mathrm{CO}_{2}$ will rise. 
Significant arterio-venous $\mathrm{CO}_{2}$ differences have been reported during cardiopulmonary arrest and resuscitation with a predominant venous respiratory acidaemia and a paradoxical arterial alkalaemia (Weil et al., 1986). The decreased pulmonary blood flow results in the accumulation of $\mathrm{CO}_{2}$ in the venous circuit, even with adequate ventilation. Carbon dioxide delivery to the lungs is readily eliminated due to the high ventilation/perfusion ratio, resulting in arterial alkalaemia. In this study, the end-tidal $\mathrm{CO}_{2}$ values were high at the commencement of CPR and in the first few minutes after return of spontaneous circulation, as a consequence of the rapid elimination of accumulated $\mathrm{CO}_{2}$ in the venous circuit. There was a significant difference between the end-tidal $\mathrm{CO}_{2}$ concentration in patients undergoing CPR before return of spontaneous circulation and patients who failed to develop spontaneous output. These results support previous observations that end-tidal $\mathrm{CO}_{2}$ values in resuscitated animals were significantly higher than in animals in which resuscitative efforts were unsuccessful suggesting that measurement of end-tidal $\mathrm{CO}_{2}$ has predictive value (Sanders et al., 1985; Grundler et al., 1986).

The concept that end-tidal $\mathrm{CO}_{2}$ reflects pulmonary blood flow has certain limitations. End-tidal $\mathrm{CO}_{2}$ values can be affected by carbon dioxide production, ventilation and pulmonary perfusion. If manual ventilation is used instead of a mechanical device, care must be taken to provide a constant ventilation rate and tidal volume. The assumption that $\mathrm{CO}_{2}$ production is constant during resuscitation may be fallacious. However, it is unlikely that $\mathrm{CO}_{2}$ production decreases immediately after cardiac arrest (Gutipati et al., 1986). End-tidal $\mathrm{CO}_{2}$ changes related to variation in carbon dioxide production appear insignificant compared with the perfusion changes. In the present study, following a 'wash-out' effect with high end-tidal $\mathrm{CO}_{2}$ values shortly after patients were established on the Thumper, endtidal $\mathrm{CO}_{2}$ concentrations decreased slightly during $\mathrm{CPR}$. Upon return of spontaneous circulation, values increased several fold. In addition, although sodium bicarbonate administration has been shown to result in acute increases in end-tidal $\mathrm{CO}_{2}$ concentration, the rise is less than that seen with return of spontaneous circulation and of much shorter duration (Falk et al., 1988; Garnett et al., 1987).

During cardiopulmonary resuscitation, an abrupt increase in end-tidal $\mathrm{CO}_{2}$ values, under conditions of constant ventilation and in the absence of recent sodium bicarbonate administration, provides the earliest evidence of return of spontaneous circulation. Therefore, chest compression need not be interrupted in order to establish whether spontaneous circulation has been restored.

We conclude that end-tidal $\mathrm{CO}_{2}$ measurement can provide a useful non-invasive monitor of blood flow produced by precordial compression during cardiac arrest.

\section{ACKNOWLEDGEMENTS}

We wish to thank Mrs E. MacDonald and Mrs C. Sinclair for preparation of the manuscript and Ohmeda for the use of the $\mathrm{CO}_{2}$ analyser. 


\section{REFERENCES}

Chamberlain D. A., Gattiker R., Hart H. N. et al. (1987) Recent achievements and present controversies in cardiopulmonary resuscitation European Heart Journal 8, 438-43.

Del Guercio L. R. M., Feins N. R., Cohn J. D. et al. (1965) Comparison of blood flow during external and internal cardiac massage in man. Circulation 32 (suppl), 171-80.

Falk J. L., Rackow E. C. \& Weil M. H. (1988) End-tidal carbon dioxide concentration during cardiopulmonary resuscitation. New England Journal of Medicine 318, 607-11.

Garnett A. R., Ornato J. P., Gonzalez E. R. \& Johnson E. B. (1987) End-tidal carbon dioxide monitoring during cardio-pulmonary resuscitation. Journal of the American Medical Association 257, 512-5.

Grundler W., Weil M. H. \& Rackow E. C. (1986) Arterial venous carbon dioxide and pH gradients during cardiac arrest. Circulation 74, 1071-4.

Gutipati C. V., Weil M. H., Deshmukh H. et al. (1986) Critical reduction in oxygen transport without reduction in oxygen consumption in experimental cardiopulmonary resuscitation. Critical Care Medicine 14, 432.

Little K., Auchincloss J. M. \& Reaves C. S. (1974) A mechanical cardio-pulmonary life support system. Resuscitation 3, 63-8.

Powles A. C. P. \& Campbell E. G. M. (1978) An improved re-breathing method for measuring venous carbon dioxide tension and its clinical application. Canadian Medical Association Journal 118, 501-4.

Sanders A. B., Ewy G. A., Bragg S. et al. (1985) Expired PCO2 as a prognostic indicator of successful 을 resuscitation from cardiac arrest. Annals of Emergency Medicine 14, 948-52.

Skinner D. V. (1989) Monitoring resuscitation. British Medical Journal, 298, 1597.

UK Resuscitation Council (1984) Cardio-pulmonary resuscitation. Laerdal Medical Limited, London.

Weil M. H., Bisera J., Trevino R. P. et al. (1985) Cardiac output and end-tidal carbon dioxide. Critical Care Medicine 13, 907-9.

Weil M. H., Rackow E. C., Trevino R. et al. (1986) Differences in acid-based state between venous ang arterial blood during cardio-pulmonary resuscitation. New England Journal of Medicine, 315, 153-6. 